\title{
Review: patients with infectious mononucleosis have long lasting fatigue, and poor physical functioning predicts delayed recovery
}

\author{
Candy B, Chalder T, Cleare AJ, et al. Recovery from infectious mononucleosis: a case for more than symptomatic therapy? A \\ systematic review. Br J Gen Pract 2002;52:844-51.
}

\section{QUESTION: In patients with infectious mononucleosis (IM), how frequent are chronic symptoms? Are any risk factors associated with chronic symptoms? Which interventions, if any, are preventive?}

\section{Data sources}

Studies were identified by searching Medline, EMBASE/ Excerpta Medica, PsycINFO, and CINAHL (to October 2001) and the Science and Social Sciences citation indices (to November 2001). The references of retrieved studies were checked.

\section{Study selection}

English language studies were selected if they were cohort or intervention studies that explored the course of illness for $>6$ weeks after onset in uncomplicated IM.

\section{Data extraction}

A standard form was used to extract data on study design and methods, setting, patient characteristics, study purpose, definition of IM, ill health and recovery, predictors of delayed recovery, and main study outcomes.

For correspondence:

Dr M Hotopf, Guy's

King's and St Thomas'

School of Medicine,

London, UK

M.hotopf@iop.kcl.ac.uk retrospective cohort studies) were included. Sample sizes ranged from 25-337 patients, and follow up ranged from 2 months to 2 years. 5 studies reported $<80 \%$ follow up and are not included in this abstract. 5 studies examined the course of ill health. In 1 prospective study, patients recovering from IM were more likely than those with upper respiratory tract infections (URTIs) to have fatigue $(40 \%$ v $15 \%)$, hypersomnia $(22 \% v 2 \%)$, or chronic fatigue syndrome $(9-22 \% v 0-6 \%)$ at 6 months. A quasi-RCT reported that fatigue was the longest lasting symptom; $10 \%$ of patients reported not being recovered by 6 weeks. 1 retrospective study reported patients with continued symptoms at 6 months $(56 \%)$ and 11 months $(16 \%)$ with fatigue as the most persistent symptom; in another retrospective cohort study, only $1.5 \%$ of patients at a student health clinic had fatigue that persisted 2 months after onset. In 1 prospective study, psychiatric disorders were increased at onset but not at 6 months. A small retrospective study reported increased depression ( $40 \%$ absolute increase) and anxiety ( $10 \%$ absolute increase) in women 12 months after infection, but no increase in men was seen.

2 of 4 studies found no association between clinical features and poor outcome. Delayed recovery was predicted by splenomegaly at onset in 1 study ( 1 study found no association). Cervical lymphadenopathy was associated with fatigue syndrome at 1 and 2 months (1 study). In 4 studies of psychological predictors, a history of depression was not associated with poor outcome (1 study); social adversity predicted psychiatric diagnosis at 2 and 6 months ( 1 study); psychological comorbidity was associated with delayed recovery at 6 months (1 retrospective study); and a premorbid mood disorder predicted fatigue syndrome at 6 months ( 1 study). In 2 studies of demographic predictors, female sex was associated with psychological distress in recovery (1 study); 1 study showed that sex did not predict poor outcome. In 4 studies of behavioural variables, predictors for delayed recovery included bed rest (1 quasi-RCT), longer absence from school or work (1 retrospective study), and physical deconditioning (1 study). In 2 RCTs, acyclovir alone or combined with prednisolone did not reduce chronic symptoms.

\section{Conclusions}

In patients with infectious mononucleosis, the chronic symptom of fatigue is reported by up to one half of patients. Evidence for clinical and psychological predictors is mixed, but poor physical functioning consistently predicts delayed recovery. Drug therapy does not shorten recovery time.
Fukuda K, Straus SE, Hickie I, et al. The chronic fatigue syndrome: a comprehensive approach to its definition and study. International Chronic Fatigue Syndrome Study Group Ann Intern Med 1994:121:953-9.

Whiting P Bagnall AM, Sowden AJ, et al. Interventions for the treatment and management of chronic fatigue syndrome: a systematic review. JAMA 2001;286:1360-8. 